Article

\title{
Extremal Solutions of Generalized Caputo-Type Fractional-Order Boundary Value Problems Using Monotone Iterative Method
}

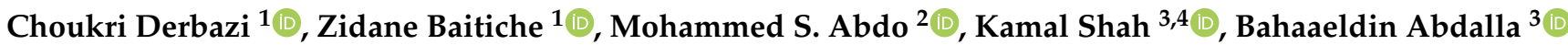 \\ and Thabet Abdeljawad 3,5,*iD
}

1 Laboratoire Equations Différentielles, Department of Mathematics, Faculty of Exact Sciences, Frères Mentouri University Constantine 1, Ain El Bey Way, P.O. Box 325, Constantine 25017, Algeria; choukri.derbazi@umc.edu.dz (C.D.); zidane.baitiche@umc.edu.dz (Z.B.)

2 Department of Mathematics, College of Education, Hodeidah University, P.O. Box 3114, Al-Hudaydah 207416, Yemen; msabdo@hoduniv.net.ye

3 Department of Mathematics and Sciences, Prince Sultan University, P.O. Box 66833, Riyadh 11586, Saudi Arabia; kshah@psu.edu.sa (K.S.); babdallah@psu.edu.sa (B.A.)

4 Department of Mathematics, University of Malakand, P.O. Box 18000, Chakdara 18800, Dir (L), Khyber Pakhtankhawa, Pakistan

5 Department of Medical Research, China Medical University, Taichung 40402, Taiwan

* Correspondence: tabdeljawad@psu.edu.sa

check for updates

Citation: Derbazi, C.; Baitiche, Z.; Abdo, M.S.; Shah, K.; Abdalla, B.; Abdeljawad, T. Extremal Solutions of Generalized Caputo-Type FractionalOrder Boundary Value Problems Using Monotone Iterative Method. Fractal Fract. 2022, 6, 146. https:// doi.org/10.3390/fractalfract6030146 Academic Editors: Asifa Tassaddiq and Muhammad Yaseen

Received: 6 February 2022

Accepted: 3 March 2022

Published: 7 March 2022

Publisher's Note: MDPI stays neutral with regard to jurisdictional claims in published maps and institutional affiliations.

Copyright: (c) 2022 by the authors. Licensee MDPI, Basel, Switzerland. This article is an open access article distributed under the terms and conditions of the Creative Commons Attribution (CC BY) license (https:// creativecommons.org/licenses/by/ $4.0 /)$.

\begin{abstract}
The aim of this research work is to derive some appropriate results for extremal solutions to a class of generalized Caputo-type nonlinear fractional differential equations (FDEs) under nonlinear boundary conditions (NBCs). The aforesaid results are derived by using the monotone iterative method, which exercises the procedure of upper and lower solutions. Two sequences of extremal solutions are generated in which one converges to the upper and the other to the corresponding lower solution. The method does not need any prior discretization or collocation for generating the aforesaid two sequences for upper and lower solutions. Further, the aforesaid techniques produce a fruitful combination of upper and lower solutions. To demonstrate our results, we provide some pertinent examples.
\end{abstract}

Keywords: $\vartheta$-Caputo derivative; extremal solutions; monotone iterative method; sequences

\section{Introduction}

Over the last few decades, fractional calculus has attracted the attention of many researchers in the community of science and technology. This is because of its significant applications in different fields of science and engineering such as mathematics, physics, chemistry, biology, economics, finance, rheology, etc. (for more details, see [1-3]). Further, the most important applications of fractional calculus can be found in the description of memory and hereditary processes. The mentioned processes can be more nicely explained by the concept of fractional-order derivatives as compared to traditional ones. Keeping their importance in mind, researchers have given much attention to the use of fractional-order derivatives and integrals in the mathematical modeling of real-world processes instead of classical derivatives and integrals. In this regard, several monographs, and plenty of papers and books have been published, in which various kinds of important results and applications have been reported. Some of these can be found in [4-7]. Nevertheless, the application of the aforesaid area has been traced out just in the last two decades. This is due to the progress in the area of chaos that revealed refined relationships with the concepts of fractional calculus. In addition, in recent times, the application of the theory of fractional calculus to robotics has opened promising aspects for future developments, where in these robots, joint-level control is usually planted by using PID-like schemes with position feedback. For instance, one famous machine using the fractional $P D^{\alpha}$ controller is known as the Hexapod robot (see [8]). Further, some important applications of fractional calculus 
can be found about the dynamics in the trajectory control of redundant manipulators, where fractional-order derivatives have a more precise appearance than classical ones (see [9]). Further, the fractional derivative is a global operator that preserves greater degrees of freedom as compared to integer-order derivative, because a classical derivative with integer-order is a local operator. It is estimated that the fractional-order derivative operation contains some types of boundary conditions that involve information on the function further out. Some researchers have proved that fractional-order derivatives play a significant role in electrochemical analysis to elucidate the mechanistic behavior of the concentration of a substrate at the electrode surface to the current. Some researchers have proved that fractional-order modelers of contaminant flow in heterogeneous porous media involving fractional derivatives are more powerful than classical ones (see [10]). It should be kept in mind that fractional-order derivative operation of a function produces a complete spectrum or accumulation, which preserves the corresponding integer-order counterpart as a special case.

It is interesting that fractional-order derivatives have not yet been uniquely defined. Various renowned mathematicians have given their own definitions. Among the said definitions, some of them have gained much more popularity and proper attention from researchers, such as Riemann-Liouville (RL), Caputo, Hilfer, Caputo-Hadamard, CaputoKatugampola, etc. It is interesting that aside from the aforesaid operators various other variants that contain singular kernels have been introduced recently. Hence the fractional differential operators have been divided into two classes including singular and non-singular. Here we state that this partition is not bad but provides a great degree of freedom in the choice of operator for the description of a particular phenomenon. It is remarkable that nearly all mentioned operators preserve memory in their respective kernels. Further, the two partitions of singular and non-singular kernels have their own benefits and drawbacks.

We remark that Hilfer-type fractional calculus unifies the aforesaid definitions. It is important that $\vartheta$-Hilfer operators constitute a wide class of fractional derivatives (FDs). In this respect, some frequent results involving $\vartheta$-FDs have been reported in [11-13]. However, various strategies exist in the literature to handle such types of problems of FDEs for computation of their solutions. Numerous tools and theories have been established so far. Iterative techniques of various kinds have key importance to investigate the aforementioned area. Among them are the monotone iterative algorithm, along with the upper and lower solutions method [14,15], fixed-point technique [16-18], and coincidence degree theory $[19,20]$. In particular, the monotone iterative method together with the technique of upper and lower solutions is an advantageous and effective tool for the existence as well as the approximation of solutions for nonlinear problems. In this regard, very useful results have been published so far. Among the iterative techniques, those introduced by Ladde, Lakshmikantham, and Vatsala [21] in 1985 for nonlinear differential equations have gained proper attention. Therefore, monotone iterative techniques associated with upper and lower solutions have been extensively used for nonlinear partial differential equations in the last few decades. In this regard, plenty of work has been published to date; a few can be found in [22-25]. Proposals have been made for classical differential equations for the first time [14,15,26-29]. In addition, the aforesaid techniques have been widely used to deal with FDEs subject to initial and boundary conditions. Some significant results can be found in [25,30-37]. We demonstrate that the said method is well known because it not only produces constructive proof for existence theorems but it also yields various comparison results, which are powerful tools to investigate the qualitative properties of solutions. Further, the sequence of iterations has useful behavior in the computation of numerical solutions to various boundary value and initial boundary value problems of classical as well FDEs. In addition, the method of upper and lower solutions is very useful for the construction of Lyapunov functions. Construction of such functions is increasingly used to derive various stability theories in dynamical systems. 
Motivated by the work cited above, and particularly by the work in [31], we determine the existence criteria of extremal solutions for the following $\vartheta$-Caputo-type FDE in a Caputo sense with NBCs

$$
\left\{\begin{array}{l}
{ }^{c} \mathbb{D}_{a^{+}}^{v ; \vartheta} \phi(\varsigma)=\mathbb{F}(\varsigma, \phi(\varsigma)), \varsigma \in \mathrm{J}:=[a, b], \\
\mathbb{G}(\phi(a), \phi(b))=0,
\end{array}\right.
$$

where $0<v \leq 1,{ }^{c} \mathbb{D}_{a^{+}}^{v ; \vartheta}$ is the $\vartheta$-fractional operator of order $v$ in the Caputo sense and this is investigated. Further, $\mathbb{F} \in C(\mathrm{~J} \times \mathbb{R}, \mathbb{R}), \mathbb{G} \in C\left(\mathbb{R}^{2}, \mathbb{R}\right)$.

The considered problem (1) in the current article includes a wide range of nonlinear FDEs involving the standard Caputo operator (for $\vartheta(\varsigma)=\varsigma$ ), Caputo-Hadamard (for $\vartheta(\varsigma)=\log \varsigma$ ), and Caputo-Katugampola (for $\vartheta(\varsigma)=\varsigma^{\mathrm{p}}, \mathrm{p}>0$ ). Further, fractional operators have been listed in Almeida [11] for further applications. Further, results acquired in the current article include the results of Franco et al. [26] if $a \rightarrow 0, \vartheta(\varsigma) \rightarrow \varsigma$, and $v=1$ as a special case.

In this regard, we also point out some recent and similar findings that used operators on many fractional problems; see [38-40]. To the best of our knowledge in this regard, no one has considered the monotone iterative procedure to obtain the existence of extremal solutions involving a $\vartheta$-Caputo derivative subject to NBCs. Therefore, motivated by the aforesaid gap, we have conducted this study.

The rest of this article is organized as follows. In Section 2, we insert some basic definitions and important results. Section 3 is devoted to studying the existence of extremal solutions for (1). In Section 4, we give two appropriate examples to highlight the feasibility of our abstract results.

\section{Basic Results}

Some fundamental results about the $\vartheta$-Caputo derivative and integral that are needed throughout this work are given below.

The function $\vartheta \in C(J, \mathbb{R})$ is non-decreasing differentiable with argument $0<\vartheta^{\prime}(\varsigma)$, at every point of $\mathrm{J}$.

Definition $1([6,11])$. The $\vartheta-R L$ fractional integral of order $v>0$ for an integrable function $\phi: \mathrm{J} \longrightarrow \mathbb{R}$ is given by

$$
\mathbb{I}_{a^{+}}^{v ; \vartheta} \phi(\varsigma)=\frac{1}{\Gamma(v)} \int_{a}^{\varsigma} \vartheta^{\prime}(s)(\vartheta(\varsigma)-\vartheta(s))^{v-1} \phi(s) \mathrm{d} s, \varsigma>a
$$

Definition 2 ([11]). Let $\vartheta, \phi \in C^{n}(\mathrm{~J}, \mathbb{R})$. The $\vartheta$-RL derivative of fractional order of a function $\phi$ with $n-1<v<n$ is given by

$$
\begin{aligned}
\mathbb{D}_{a^{+}}^{v ; \vartheta} \phi(\varsigma) & =\left(\frac{D_{t}}{\vartheta^{\prime}(\varsigma)}\right)^{n} \mathbb{I}_{a^{+}}^{n-v ; \vartheta} \phi(\varsigma) \\
& =\frac{1}{\Gamma(n-v)}\left(\frac{D_{t}}{\vartheta^{\prime}(\varsigma)}\right)^{n} \int_{a}^{\varsigma} \vartheta^{\prime}(s)(\vartheta(\varsigma)-\vartheta(s))^{n-v-1} \phi(s) \mathrm{d} s,
\end{aligned}
$$

where $n=[v]+1(n \in \mathbb{N})$, and $D_{t}=\frac{d}{d t}$.

Definition 3 ([11]). Let $\vartheta, \phi \in C^{n}(\mathrm{~J}, \mathbb{R})$. The $\vartheta$-Caputo derivative of fractional order of function $\phi$ with $n-1<v<n$ is defined by

$$
{ }^{c} \mathbb{D}_{a^{+}}^{v ; \vartheta} \phi(\varsigma)=\mathbb{I}_{a^{+}}^{n-v ; \vartheta} \phi_{\vartheta}^{[n]}(\varsigma),
$$

where $\phi_{\vartheta}^{[n]}(\varsigma)=\left(\frac{D_{\zeta}}{\vartheta^{\prime}(\varsigma)}\right)^{n} \phi(\varsigma), n=[v]+1$ for $v \notin \mathbb{N}$, and $n=v$ for $v \in \mathbb{N}$. 
One has

$$
{ }^{c} \mathbb{D}_{a^{+}}^{v ; \vartheta} \phi(\varsigma)=\left\{\begin{array}{cc}
\int_{a}^{\varsigma} \frac{\vartheta^{\prime}(s)(\vartheta(\varsigma)-\vartheta(s))^{n-v-1}}{\Gamma(n-v)} \phi_{\vartheta}^{[n]}(s) \mathrm{d} s & , \text { if } v \notin \mathbb{N}, \\
\phi_{\vartheta}^{[n]}(\varsigma) & , \text { if } v \in \mathbb{N} .
\end{array}\right.
$$

Lemma 1 ([11]). Let $v, \mu>0$, and $\phi \in C(\mathrm{~J}, \mathbb{R})$, for every $\varsigma \in \mathrm{J}$

1. ${ }^{c} \mathbb{D}_{a^{+}}^{v ; \vartheta} \mathbb{I}_{a^{+}}^{v ; \vartheta} \phi(\varsigma)=\phi(\varsigma)$,

2. $\quad \mathbb{I}_{a^{+}}^{v ; \vartheta^{+}} \mathbb{D}_{a^{+}}^{v ; \vartheta} \phi(\varsigma)=\phi(s)-\phi(a), \quad 0<v \leq 1$,

3. $\mathbb{I}_{a^{+}}^{v ; \vartheta}(\vartheta(\varsigma)-\vartheta(a))^{\mu-1}=\frac{\Gamma(\mu)}{\Gamma(\mu+v)}(\vartheta(\varsigma)-\vartheta(a))^{\mu+v-1}$,

4. ${ }^{c} \mathbb{D}_{a^{+}}^{v ; \vartheta}(\vartheta(\varsigma)-\vartheta(a))^{\mu-1}=\frac{\Gamma(\mu)}{\Gamma(\mu-v)}(\vartheta(\varsigma)-\vartheta(a))^{\mu-v-1}$,

5. ${ }^{c} \mathbb{D}_{a^{+}}^{v ; \vartheta}(\vartheta(s)-\vartheta(a))^{k}=0, \forall k<n \in \mathbb{N}$.

Definition 4 ([7]). One- and two-parameter Mittag-Leffler functions (MLFs) are recalled as

$$
\mathbb{E}_{v}(z)=\sum_{k=0}^{\infty} \frac{z^{k}}{\Gamma(v k+1)}, \quad(z \in \mathbb{R}, v>0),
$$

and

$$
\mathbb{E}_{v, \mu}(z)=\sum_{k=0}^{\infty} \frac{z^{k}}{\Gamma(v k+\mu)}, v, \mu>0 \text { and } z \in \mathbb{R},
$$

respectively. Clearly $\mathbb{E}_{1,1}(z)=\mathbb{E}_{1}(z)=\exp (z)$

Further properties of MLFs are given below.

Lemma 2 ([41]). Let $v \in(0,1)$ and $z \in \mathbb{R}$, one has

1. $\mathbb{E}_{v, 1}$ and $\mathbb{E}_{v, v}$ are non-negative.

2. $\mathbb{E}_{v, 1}(z) \leq 1, \mathbb{E}_{v, v}(z) \leq \frac{1}{\Gamma(v)}$, for any $z<0$.

For further analysis, we recall the following Lemma [31] as:

Lemma 3 ([31] (Lemma 4)). Let $v \in(0,1], \lambda \in \mathbb{R}$ and $h \in C(\mathrm{~J}, \mathbb{R})$, then, the linear version

$$
\left\{\begin{array}{l}
{ }^{c} \mathbb{D}_{a^{+}}^{v ; \vartheta} \phi(\varsigma)+\lambda \phi(\varsigma)=h(\varsigma), \varsigma \in \mathrm{J} \\
\phi(a)=\phi_{a}
\end{array}\right.
$$

has a unique solution that is described as

$$
\begin{aligned}
\phi(\varsigma) & =\theta_{a} \mathbb{E}_{v, 1}\left(-\lambda(\vartheta(\varsigma)-\vartheta(a))^{v}\right) \\
& +\int_{a}^{\varsigma} \vartheta^{\prime}(s)(\vartheta(\varsigma)-\vartheta(s))^{v-1} \mathbb{E}_{v, v}\left(-\lambda(\vartheta(\varsigma)-\vartheta(s))^{v}\right) h(s) \mathrm{d} s,
\end{aligned}
$$

where $\mathbb{E}_{v, \mu}(\cdot)$ is the two-parametric MLF defined earlier.

The given comparison results comprise a central rule in the following analysis.

Lemma 4 ([31] (Lemma 5)). Let $v \in(0,1]$, and $\lambda \in \mathbb{R}$, if $\gamma \in C(\mathrm{~J}, \mathbb{R})$ obey the given relation

$$
\left\{\begin{array}{l}
{ }^{c} \mathbb{D}_{a^{+}}^{v ; \vartheta} \gamma(\varsigma) \geq-\lambda \gamma(\varsigma), \quad \varsigma \in(a, b] \\
\gamma(a) \geq 0
\end{array}\right.
$$

then $\gamma(\varsigma) \geq 0$, for all $\varsigma \in \mathrm{J}$. 


\section{Main Results}

Here, key findings are established about the $\vartheta$-Caputo FDEs (1). We develop two monotone iterative sequences for upper and lower solutions, respectively.

Definition 5. The function $\phi \in C(\mathrm{~J}, \mathbb{R})$ such that ${ }^{c} \mathrm{D}_{a^{+}}^{v ; \vartheta} \phi$ exists and is continuous on $\mathrm{J}$ and is known to be a solution of the problem (1). Further, $\phi$ gives the statistics of the equation ${ }^{c} \mathbb{D}_{a^{+}}^{v ; \vartheta} \phi(\varsigma)=$ $\mathbb{F}(\varsigma, \phi(\varsigma))$, for each $\varsigma \in \mathrm{J}$ and the $\mathrm{NBC} s$

$$
\mathbb{G}(\phi(a), \phi(b))=0 .
$$

Subsequently, we mention the definitions of extremal solutions of $\vartheta$-Caputo FDEs (1).

Definition 6. The mapping $\phi_{0} \in C(\mathrm{~J}, \mathbb{R})$ is known as lower solution (1), if it satisfies

$$
\left\{\begin{array}{l}
{ }^{c} \mathbb{D}_{a^{+}}^{v ; \vartheta} \phi_{0}(\varsigma) \leq \mathbb{F}\left(\varsigma, \phi_{0}(\varsigma)\right), \quad t \in(a, b], \\
\mathbb{G}\left(\phi_{0}(a), \phi_{0}(b)\right) \leq 0 .
\end{array}\right.
$$

An upper solution $\omega_{0} \in C(\mathrm{~J}, \mathbb{R})$ of the problem (1) can be defined in a similar way by reversing the above inequality.

Now to move forward, we will introduce the following conditions:

Hypothesis 1. There exist $\phi_{0}$ and $\omega_{0}$ as lower and upper solutions of problem (1) in $C(\mathrm{~J}, \mathbb{R})$ respectively, with $\phi_{0}(\varsigma) \leq \omega_{0}(\varsigma), \varsigma \in \mathrm{J}$.

Hypothesis 2. There exists a constant $\lambda>0$ with

$$
\mathbb{F}(\varsigma, \mathrm{y})-\mathbb{F}(\varsigma, \mathrm{x}) \geq-\lambda(\mathrm{y}-\mathrm{x}) \text { for } \phi_{0}(\varsigma) \leq \mathrm{x} \leq \mathrm{y} \leq \omega_{0}(\varsigma), \varsigma \in \mathrm{J} .
$$

Hypothesis 3. There exist constants $c>0$ and $d \geq 0$ with $\phi_{0}(a) \leq u_{1} \leq u_{2} \leq \omega_{0}(a)$, $\phi_{0}(b) \leq v_{1} \leq v_{2} \leq \omega_{0}(b)$, such that

$$
\mathbb{G}\left(u_{2}, v_{2}\right)-\mathbb{G}\left(u_{1}, v_{1}\right) \leq c\left(u_{2}-u_{1}\right)-d\left(v_{2}-v_{1}\right),
$$

Now, we shall apply the monotonous method to prove our key findings.

Theorem 1. Let $\mathbb{F}: \mathrm{J} \times \mathbb{R} \longrightarrow \mathbb{R}$ be continuous. Assume that Hypotheses 1-3 hold. Then there exist two monotone iterative sequences $\left\{\phi_{n}\right\}$ and $\left\{\omega_{n}\right\}$, which are converging uniformly on $\mathrm{J}$ to the extremal solutions of (1) in the sector $\left[\phi_{0}, \omega_{0}\right]$, where

$$
\left[\phi_{0}, \omega_{0}\right]=\left\{z \in C(\mathrm{~J}, \mathbb{R}): \phi_{0}(\varsigma) \leq z(\varsigma) \leq \omega_{0}(\varsigma), \quad \varsigma \in \mathrm{J}\right\} .
$$

Proof. First, for any $\phi_{0}(\varsigma), \omega_{0}(\varsigma) \in C(\mathrm{~J}, \mathbb{R})$ and $\lambda>0$, we consider the following FDEs

$$
\left\{\begin{array}{l}
{ }^{c} \mathbb{D}_{a^{+}}^{v ; \vartheta} \phi_{n+1}(\varsigma)=\mathbb{F}\left(\varsigma, \phi_{n}(\varsigma)\right)-\lambda\left(\phi_{n+1}(\varsigma)-\phi_{n}(\varsigma)\right), \quad \varsigma \in \mathrm{J}, \\
\phi_{n+1}(a)=\phi_{n}(a)-\frac{1}{c} \mathbb{G}\left(\phi_{n}(a), \phi_{n}(b)\right),
\end{array}\right.
$$

and

$$
\left\{\begin{array}{l}
{ }^{c} \mathbb{D}_{a^{+}}^{v ; \vartheta} \omega_{n+1}(\varsigma)=\mathbb{F}\left(\varsigma, \omega_{n}(\varsigma)\right)-\lambda\left(\omega_{n+1}(\varsigma)-\omega_{n}(\varsigma)\right), \quad \varsigma \in \mathrm{J}, \\
\omega_{n+1}(a)=\omega_{n}(a)-\frac{1}{c} \mathbb{G}\left(\omega_{n}(a), \omega_{n}(b)\right)
\end{array}\right.
$$


According to Lemma 3, one can deduce that (2) and (3) preserve at most one solution in $C(\mathrm{~J}, \mathbb{R})$. Thus we have

$$
\begin{aligned}
& \phi_{n+1}(\varsigma)=\left(\phi_{n}(a)-\frac{1}{c} \mathbb{G}\left(\phi_{n}(a), \phi_{n}(b)\right)\right) \mathbb{E}_{v, 1}\left(-\lambda(\vartheta(\varsigma)-\vartheta(a))^{v}\right) \\
& +\int_{a}^{\varsigma} \vartheta^{\prime}(s)(\vartheta(s)-\vartheta(s))^{v-1} \mathbb{E}_{v, v}\left(-\lambda(\vartheta(s)-\vartheta(s))^{v}\right)\left(\mathbb{F}\left(s, \phi_{n}(s)\right)+\lambda \phi_{n}(s)\right) \mathrm{d} s, \varsigma \in \mathrm{J}, \\
& \omega_{n+1}(\varsigma)=\left(\omega_{n}(a)-\frac{1}{c} \mathbb{G}\left(\omega_{n}(a), \omega_{n}(b)\right)\right) \mathbb{E}_{v, v}\left(-\lambda(\vartheta(s)-\vartheta(a))^{v}\right) \\
& +\int_{a}^{\varsigma} \vartheta^{\prime}(s)(\vartheta(\varsigma)-\vartheta(s))^{v-1} \mathbb{E}_{v, v}\left(-\lambda(\vartheta(s)-\vartheta(s))^{v}\right)\left(\mathbb{F}\left(s, \omega_{n}(s)\right)+\lambda \omega_{n}(s)\right) \mathrm{d}, \varsigma \in \mathrm{J} .
\end{aligned}
$$

For appropriateness, the proof will be divided into a number of steps.

Step 1: The sequences $\phi_{n}(\zeta), \omega_{n}(\zeta)(n \geq 1)$ are lower and upper solutions of (1), correspondingly. Moreover, we assume

$$
\phi_{0}(\varsigma) \leq \phi_{1}(\varsigma) \leq \cdots \leq \phi_{n}(\varsigma) \leq \cdots \leq \omega_{n}(\varsigma) \leq \cdots \leq \omega_{1}(\varsigma) \leq \omega_{0}(\varsigma), \quad \varsigma \in \mathrm{J}
$$

Firstly, we show that

$$
\phi_{0}(\varsigma) \leq \phi_{1}(\varsigma) \leq \omega_{1}(\varsigma) \leq \omega_{0}(\varsigma), \quad \varsigma \in \mathrm{J}
$$

Set $\gamma(\varsigma)=\phi_{1}(\varsigma)-\phi_{0}(\varsigma)$. From (2) and Definition 6, we obtain

$$
\begin{aligned}
{ }^{c} \mathbb{D}_{a^{+}}^{v ; \vartheta} \gamma(\varsigma) & ={ }^{c} \mathbb{D}_{a^{+}}^{v ; \vartheta} \phi_{1}(\varsigma)-{ }^{c} \mathbb{D}_{a^{+}}^{v ; \vartheta} \phi_{0}(\varsigma) \\
& \geq \mathbb{F}\left(\varsigma, \phi_{0}(\varsigma)\right)-\lambda\left(\phi_{1}(\varsigma)-\phi_{0}(\varsigma)\right)-\mathbb{F}\left(\varsigma, \phi_{0}(\varsigma)\right) \\
& =-\lambda \gamma(\varsigma) .
\end{aligned}
$$

Again, since $\gamma(a)=-\frac{1}{c} \mathbb{G}\left(\phi_{0}(a), \phi_{0}(b)\right) \geq 0, \gamma(\varsigma) \geq 0$, for $\varsigma \in \mathrm{J}$ due to Lemma 4 . Thus, $\phi_{0}(\varsigma) \leq \phi_{1}(\varsigma)$.

Similarly, we can find that $\omega_{1}(\varsigma) \leq \omega_{0}(\varsigma), \varsigma \in \mathrm{J}$.

Now, let $\gamma(\varsigma)=\omega_{1}(\varsigma)-\phi_{1}(\varsigma)$. Using (2) and (3) together with Hypotheses 2 and 3 , we obtain

$$
\begin{aligned}
{ }^{c} \mathbb{D}_{a^{+}}^{v ; \vartheta} \gamma(\varsigma) & =\mathbb{F}\left(\varsigma, \omega_{0}(\varsigma)\right)-\mathbb{F}\left(\varsigma, \phi_{0}(\varsigma)\right)-\lambda\left(\omega_{1}(\varsigma)-\omega_{0}(\varsigma)\right)+\lambda\left(\phi_{1}(\varsigma)-\phi_{0}(\varsigma)\right) \\
& \geq-\lambda\left(\omega_{0}(\varsigma)-\phi_{0}(\varsigma)\right)-\lambda\left(\omega_{1}(\varsigma)-\omega_{0}(\varsigma)\right)+\lambda\left(\phi_{1}(\varsigma)-\phi_{0}(\varsigma)\right) \\
& =-\lambda \gamma(\varsigma) .
\end{aligned}
$$

Since

$$
\begin{aligned}
\gamma(a) & =\left(\omega_{0}(a)-\phi_{0}(a)\right)-\frac{1}{c}\left(\mathbb{G}\left(\omega_{0}(a), \omega_{0}(b)\right)-\mathbb{G}\left(\phi_{0}(a), \phi_{0}(b)\right)\right) \\
& \geq \frac{d}{c}\left(\omega_{0}(b)-\phi_{0}(b)\right) \geq 0
\end{aligned}
$$

we obtain $\phi_{1}(\varsigma) \leq \omega_{1}(\varsigma), \varsigma \in \mathrm{J}$ due to Lemma 4 .

Secondly, we show that $\phi_{1}(\varsigma), \omega_{1}(\varsigma)$ are extremum solutions of (1). Since $\phi_{0}$ and $\omega_{0}$ are lower and upper solutions of (1), by Hypotheses 2 and 3, we obtain

$$
{ }^{c} \mathbb{D}_{a^{+}}^{v ; \vartheta} \phi_{1}(\varsigma)=\mathbb{F}\left(\varsigma, \phi_{0}(\varsigma)\right)-\lambda\left(\phi_{1}(\varsigma)-\phi_{0}(\varsigma)\right) \leq \mathbb{F}\left(\varsigma, \phi_{1}(\varsigma)\right),
$$

and

$$
\begin{aligned}
\mathbb{G}\left(\phi_{1}(a), \phi_{1}(b)\right) & \leq \mathbb{G}\left(\phi_{0}(a), \phi_{0}(b)\right)+c\left(\phi_{1}(a)-\phi_{0}(a)\right)-d\left(\phi_{1}(b)-\phi_{0}(b)\right) \\
& =-d\left(\phi_{1}(b)-\phi_{0}(b)\right) \\
& \leq 0
\end{aligned}
$$


Therefore, $\phi_{1}(\varsigma)$ is a lower solution of (1). Analogously, it is obvious that $\omega_{1}(\varsigma)$ is an upper solution of (1).

Through the above debates and induction, we can show that $\phi_{n}(\varsigma), \omega_{n}(\varsigma),(n \geq 1)$ are lower and upper solutions of (1), respectively, and the assumption (4) is true.

Step 2: $\phi_{n} \rightarrow \phi$ and $\omega_{n} \rightarrow \omega$.

First, we prove that $\left\{\phi_{n}\right\}$ is uniformly bounded. By considering supposition Hypothesis 2, we may write

$$
\mathbb{F}\left(\varsigma, \phi_{0}(\varsigma)\right)+\lambda \phi_{0}(\varsigma) \leq \mathbb{F}\left(\zeta, \phi_{n}(\varsigma)\right)+\lambda \phi_{n}(\varsigma) \leq \mathbb{F}\left(\varsigma, \omega_{0}(\varsigma)\right)+\lambda \omega_{0}(\varsigma), \quad \varsigma \in \mathrm{J}
$$

i.e.,

$$
\begin{aligned}
0 & \leq \mathbb{F}\left(\varsigma, \phi_{n}(\varsigma)\right)-\mathbb{F}\left(\varsigma, \phi_{0}(\varsigma)\right)+\lambda\left(\phi_{n}(\varsigma)-\phi_{0}(\varsigma)\right) \\
& \leq \mathbb{F}\left(\varsigma, \omega_{0}(\varsigma)\right)-\mathbb{F}\left(\varsigma, \phi_{0}(\varsigma)\right)+\lambda\left(\omega_{0}(\varsigma)-\phi_{0}(\varsigma)\right) .
\end{aligned}
$$

Hence, we obtain

$$
\begin{aligned}
\left|\mathbb{F}\left(\varsigma, \phi_{n}(\varsigma)\right)-\mathbb{F}\left(\varsigma, \phi_{0}(\varsigma)\right)+\lambda\left(\phi_{n}(\varsigma)-\phi_{0}(\varsigma)\right)\right| & \leq \mid \mathbb{F}\left(\varsigma, \omega_{0}(\varsigma)\right)-\mathbb{F}\left(\varsigma, \phi_{0}(\varsigma)\right) \\
& +\lambda\left(\omega_{0}(\varsigma)-\phi_{0}(\varsigma)\right) \mid .
\end{aligned}
$$

Thus

$$
\begin{aligned}
\left|\mathbb{F}\left(\varsigma, \phi_{n}(\varsigma)\right)+\lambda \phi_{n}(\varsigma)\right| & \leq\left|\mathbb{F}\left(\varsigma, \phi_{n}(\varsigma)\right)-\mathbb{F}\left(\varsigma, \phi_{0}(\varsigma)\right)+\lambda\left(\phi_{n}(\varsigma)-\phi_{0}(\varsigma)\right)\right| \\
& +\left|\mathbb{F}\left(\varsigma, \phi_{0}(\varsigma)\right)+\lambda \phi_{0}(\varsigma)\right| \\
& \leq\left|\mathbb{F}\left(\varsigma, \omega_{0}(\varsigma)\right)-\mathbb{F}\left(\varsigma, \phi_{0}(\varsigma)\right)+\lambda\left(\omega_{0}(\varsigma)-\phi_{0}(\varsigma)\right)\right| \\
& +\left|\mathbb{F}\left(\varsigma, \phi_{0}(\varsigma)\right)+\lambda \phi_{0}(\varsigma)\right| \\
& \leq 2\left|\mathbb{F}\left(\varsigma, \phi_{0}(\varsigma)\right)+\lambda \phi_{0}(\varsigma)\right|+\left|\mathbb{F}\left(\varsigma, \omega_{0}(\varsigma)\right)+\lambda \omega_{0}(\varsigma)\right| .
\end{aligned}
$$

Since $\phi_{0}, \mathbb{F}$ are continuous on $\mathrm{J}$, we can see a constant $\mathbb{M}$ independent of $n$ with

$$
\left|\mathbb{F}\left(\zeta, \phi_{n}(\varsigma)\right)+\lambda \phi_{n}(\varsigma)\right| \leq \mathbb{M}
$$

Furthermore, from Hypothesis 3, we can obtain

$$
\phi_{0}(a)-\frac{1}{c} \mathbb{G}\left(\phi_{0}(a), \phi_{0}(b)\right) \leq \phi_{n}(a)-\frac{1}{c} \mathbb{G}\left(\phi_{n}(a), \phi_{n}(b)\right) \leq \omega_{0}(a)-\frac{1}{c} \mathbb{G}\left(\omega_{0}(a), \omega_{0}(b)\right),
$$

i.e.,

$$
\begin{aligned}
0 & \leq \phi_{n}(a)-\phi_{0}(a)-\frac{1}{c}\left(\mathbb{G}\left(\phi_{n}(a), \phi_{n}(b)\right)-\mathbb{G}\left(\phi_{0}(a), \phi_{0}(b)\right)\right) \\
& \leq \omega_{0}(a)-\phi_{0}(a)-\frac{1}{c}\left(\mathbb{G}\left(\omega_{0}(a), \omega_{0}(b)\right)-\mathbb{G}\left(\phi_{0}(a), \phi_{0}(b)\right)\right) .
\end{aligned}
$$

Hence, we obtain

$$
\begin{aligned}
& \left|\phi_{n}(a)-\phi_{0}(a)-\frac{1}{c}\left(\mathbb{G}\left(\phi_{n}(a), \phi_{n}(b)\right)-\mathbb{G}\left(\phi_{0}(a), \phi_{0}(b)\right)\right)\right| \leq \\
& \left|\omega_{0}(a)-\phi_{0}(a)-\frac{1}{c}\left(\mathbb{G}\left(\omega_{0}(a), \omega_{0}(b)\right)-\mathbb{G}\left(\phi_{0}(a), \phi_{0}(b)\right)\right)\right| \\
& \leq\left|\phi_{0}(a)-\frac{1}{c} \mathbb{G}\left(\phi_{0}(a), \phi_{0}(b)\right)\right|+\left|\omega_{0}(a)-\frac{1}{c} \mathbb{G}\left(\omega_{0}(a), \omega_{0}(b)\right)\right| .
\end{aligned}
$$


Thus

$$
\begin{aligned}
\left|\phi_{n}(a)-\frac{1}{c} \mathbb{G}\left(\phi_{n}(a), \phi_{n}(b)\right)\right| & \leq\left|\phi_{n}(a)-\phi_{0}(a)-\frac{1}{c}\left(\mathbb{G}\left(\phi_{n}(a), \phi_{n}(b)\right)-\mathbb{G}\left(\phi_{0}(a), \phi_{0}(b)\right)\right)\right| \\
& +\left|\phi_{0}(a)-\frac{1}{c} \mathbb{G}\left(\phi_{0}(a), \phi_{0}(b)\right)\right| \\
& \leq 2\left|\phi_{0}(a)-\frac{1}{c} \mathbb{G}\left(\phi_{0}(a), \phi_{0}(b)\right)\right|+\left|\omega_{0}(a)-\frac{1}{c} \mathbb{G}\left(\omega_{0}(a), \omega_{0}(b)\right)\right| .
\end{aligned}
$$

Since $\phi_{0}, \omega_{0}$ and $\mathbb{G}$ are continuous functions, we can find a constant $\mathbb{K}$ independent of $n$, such that

$$
\left|\phi_{n}(a)-\frac{1}{c} \mathbb{G}\left(\phi_{n}(a), \phi_{n}(b)\right)\right| \leq \mathbb{K} .
$$

Moreover, by (2) and (3) we have

$$
\begin{aligned}
& \left|\phi_{n+1}(s)\right|=\left|\phi_{n}(a)-\frac{1}{c} \mathbb{G}\left(\phi_{n}(a), \phi_{n}(b)\right)\right| \mathbb{E}_{v, 1}\left(-\lambda(\vartheta(s)-\vartheta(a))^{v}\right) \\
& +\int_{a}^{\varsigma} \vartheta^{\prime}(s)(\vartheta(s)-\vartheta(s))^{v-1} \mathbb{E}_{\nu, v}\left(-\lambda(\vartheta(s)-\vartheta(s))^{v}\right)\left|\mathbb{F}\left(s, \phi_{n}(s)\right)+\lambda \phi_{n}(s)\right| \mathrm{d} s, s \in \mathrm{J} .
\end{aligned}
$$

Using Lemma 2 along with (5) and (6), we obtain

$$
\begin{aligned}
\left|\phi_{n+1}(\varsigma)\right| & =\mathbb{K}+\frac{\mathbb{M}}{\Gamma(v)} \int_{a}^{\varsigma} \vartheta^{\prime}(s)(\vartheta(\varsigma)-\vartheta(s))^{v-1} \mathrm{~d} s \\
& \leq \mathbb{K}+\frac{\mathbb{M}(\vartheta(b)-\vartheta(a))^{v}}{\Gamma(v+1)} .
\end{aligned}
$$

Hence, $\left\{\phi_{n}\right\}$ is uniformly bounded in $C(\mathrm{~J}, \mathbb{R})$. With same argument one can deduce that $\left\{\omega_{n}\right\}$ is uniformly bounded.

It is necessary to derive that the sequences $\left\{\phi_{n}\right\}$ and $\left\{\omega_{n}\right\}$ are equi-continuous on J. To do this, choosing $\varsigma_{1}, \varsigma_{2} \in \mathrm{J}$, with $\varsigma_{1} \leq \varsigma_{2}$. By (5) and (6) and Lemma 2 , we have

$$
\begin{aligned}
& \left|\phi_{n+1}\left(\varsigma_{2}\right)-\phi_{n+1}\left(\varsigma_{1}\right)\right| \leq\left|\phi_{n}(a)-\frac{1}{c} \mathbb{G}\left(\phi_{n}(a), \phi_{n}(b)\right)\right| \mid \mathbb{E}_{v, 1}\left(-\lambda\left(\vartheta\left(\varsigma_{2}\right)-\vartheta(a)\right)^{v}\right)- \\
& \mathbb{E}_{v, 1}\left(-\lambda\left(\vartheta\left(\varsigma_{1}\right)-\vartheta(a)\right)^{v}\right) \mid \\
& +\int_{a}^{\varsigma_{1}} \frac{\vartheta^{\prime}(s)\left[\left(\vartheta\left(\varsigma_{1}\right)-\vartheta(s)\right)^{v-1}-\left(\vartheta\left(\varsigma_{2}\right)-\vartheta(s)\right)^{v-1}\right]}{\Gamma(v)}\left|\mathbb{F}\left(s, \phi_{n}(s)\right)+\lambda \phi_{n}(s)\right| \mathrm{d} s \\
& +\int_{\varsigma_{1}}^{\varsigma_{2}} \frac{\vartheta^{\prime}(s)\left(\vartheta\left(\varsigma_{2}\right)-\vartheta(s)\right)^{v-1}}{\Gamma(v)}\left|\mathbb{F}\left(s, \phi_{n}(s)\right)+\lambda \phi_{n}(s)\right| \mathrm{ds} \\
& \leq \mathbb{K}\left|\mathbb{E}_{v, 1}\left(-\lambda\left(\vartheta\left(\varsigma_{2}\right)-\vartheta(a)\right)^{v}\right)-\mathbb{E}_{v, 1}\left(-\lambda\left(\vartheta\left(\varsigma_{1}\right)-\vartheta(a)\right)^{v}\right)\right| \\
& +\frac{2 \mathbb{M}}{\Gamma(v+1)}\left(\vartheta\left(\varsigma_{2}\right)-\vartheta\left(\varsigma_{1}\right)\right)^{v} .
\end{aligned}
$$

By the continuity of $\mathbb{E}_{v, 1}\left(-\lambda(\vartheta(s)-\vartheta(a))^{v}\right)$ on $\mathrm{J}$, the right-hand-side of the preceding inequality approaches zero, when $\varsigma_{1} \rightarrow \varsigma_{2}$. This implies that $\left\{\phi_{n}(\varsigma)\right\}$ is equi-continuous on J. Likewise, we can demonstrate that $\left\{\omega_{n}(\zeta)\right\}$ is equi-continuous. Hence, by using Ascoli-Arzelás theorem, the sequence $\phi_{n_{k}}(\zeta) \rightarrow \phi^{*}(\varsigma)$ and $\omega_{n_{k}}(\varsigma) \rightarrow \omega^{*}(\varsigma)$ as $k \rightarrow \infty$. Hence the aforesaid relation combined under the monotonicity of sequences $\left\{\phi_{n}(\varsigma)\right\}$ and $\left\{\omega_{n}(\varsigma)\right\}$ yields

$$
\lim _{n \rightarrow \infty} \phi_{n}(\varsigma)=\phi^{*}(\varsigma) \text { and } \lim _{n \rightarrow \infty} \omega_{n}(t)=\omega^{*}(\varsigma),
$$

uniformly on $\varsigma \in \mathrm{J}$ and the limit functions $\phi^{*}, \omega^{*}$ satisfy (1).

Step 3: $\phi^{*}$ and $\omega^{*}$ are maximal and minimal solutions of $(1)$ in $\left[\phi_{0}, \omega_{0}\right]$. 
Let $z \in\left[\phi_{0}, \omega_{0}\right]$ be any solution of (1). Suppose that

$$
\phi_{n}(\varsigma) \leq z(\varsigma) \leq \omega_{n}(\varsigma), \quad \varsigma \in \mathrm{J}, \text { for some } n \in \mathbb{N}^{*} .
$$

Setting $\gamma(\varsigma)=z(\varsigma)-\phi_{n+1}(\varsigma)$. It follows that

$$
\begin{aligned}
{ }^{c} \mathbb{D}_{a^{+}}^{v ; \vartheta} \gamma(\varsigma) & =\mathbb{F}(\varsigma, z(\varsigma))-\mathbb{F}\left(\varsigma, \phi_{n}(\varsigma)\right)+\lambda\left(\phi_{n+1}(\varsigma)-\phi_{n}(\varsigma)\right) \\
& \geq-\lambda\left(z(\varsigma)-\phi_{n}(\varsigma)\right)+\lambda\left(\phi_{n+1}(\varsigma)-\phi_{n}(\varsigma)\right) \\
& =-\lambda \gamma(\varsigma) .
\end{aligned}
$$

Furthermore

$$
\begin{aligned}
\phi_{n+1}(a) & =\phi_{n}(a)-\frac{1}{c} \mathbb{G}\left(\phi_{n}(a), \phi_{n}(b)\right) \\
& =\phi_{n}(a)+\frac{1}{c} \mathbb{G}(z(a), z(b))-\frac{1}{c} \mathbb{G}\left(\phi_{n}(a), \phi_{n}(b)\right) \\
& \leq z(a)-\frac{d}{c}\left(z(b)-\phi_{n}(b)\right) \\
& \leq z(a),
\end{aligned}
$$

that is

$$
\gamma(a) \geq 0 \text {. }
$$

In view of Lemma 4 , we obtain $\gamma(\varsigma) \geq 0, \varsigma \in \mathrm{J}$, which implies

$$
\phi_{n+1}(\varsigma) \leq z(\varsigma), \varsigma \in \mathrm{J} .
$$

Utilizing the same procedure, we can derive that

$$
z(\varsigma) \leq \omega_{n+1}(\varsigma), \varsigma \in \mathrm{J} .
$$

Hence,

$$
\phi_{n+1}(\varsigma) \leq z(\varsigma) \leq \omega_{n+1}(\varsigma), \varsigma \in \mathrm{J} .
$$
one has

Hence (7) is satisfied on J for all $n \in \mathbb{N}^{*}$. Employing $n \rightarrow \infty$ on (7) from either side,

$$
\phi^{*} \leq z \leq \omega^{*} .
$$

This confirms that $\phi^{*}, \omega^{*}$ are the extremal solutions of $(1)$ in $\left[\phi_{0}, \omega_{0}\right]$.

\section{Illustrative Problems}

This section includes some test problems for the illustration of our main results.

Example 1. Consider the $\vartheta$-Caputo FDE (1) with

$$
v=0.5, a=1, b=e, \vartheta(\varsigma)=\ln \varsigma .
$$

In order to justify that Theorem 1 is valid, we take

$$
\left\{\begin{array}{l}
\mathbb{F}(\varsigma, \phi(\varsigma))=1-\phi^{2}(\varsigma)+2 \sqrt{\ln \zeta}, \quad \text { for } \varsigma \in[1, e], \\
\mathbb{G}(\phi(1), \phi(e))=\phi(1)-1 .
\end{array}\right.
$$

Without difficulty, we can infer that

$$
\phi_{0}(\varsigma)=1, \quad \omega_{0}(\varsigma)=1+\sqrt{\ln \varsigma},
$$


are lower and upper solutions of $(1)$, respectively. It is apparent that $\phi_{0}(\varsigma) \leq \omega_{0}(\varsigma)$, for $\varsigma \in[1, e]$. In addition, for $\phi_{0}(\varsigma) \leq \mathrm{x} \leq \mathrm{y} \leq \omega_{0}(\varsigma)$, we have

$$
\mathbb{F}(\varsigma, \mathrm{y})-\mathbb{F}(\varsigma, \mathrm{x}) \geq-4(\mathrm{y}-\mathrm{x}), \quad \varsigma \in[1, e] .
$$

Hence the Hypothesis 2 holds with $\lambda=4$. Further, if $\phi_{0}(1) \leq u_{1} \leq u_{2} \leq \omega_{0}(1), \phi_{0}(e) \leq$ $v_{1} \leq v_{2} \leq \omega_{0}(e)$, we have

$$
\mathbb{G}\left(u_{2}, v_{2}\right)-\mathbb{G}\left(u_{1}, v_{1}\right) \leq\left(u_{2}-u_{1}\right) .
$$

Therefore, Hypothesis 3 holds with $c=1$ and $d=0$. An application of Theorem 1 shows that the problem (1) with the data (8) and (9) has extremal solutions in the region $\left[\phi_{0}, \omega_{0}\right]$. Moreover, the monotone iterative sequences $\left\{\phi_{n}\right\}_{n \in \mathbb{N}},\left\{\omega_{n}\right\}_{n \in \mathbb{N}}$ can be acquired by

$$
\begin{aligned}
\phi_{n+1}(\varsigma) & =\mathbb{E}_{0.5,1}\left(-4 \sqrt{\ln \frac{\varsigma}{s}}\right)+\int_{1}^{\varsigma}\left(\ln \frac{\varsigma}{s}\right)^{-0.5} \mathbb{E}_{0.5,0.5}\left(-4 \sqrt{\ln \frac{\varsigma}{s}}\right) \\
& \times\left(1-\phi_{n}^{2}(s)+2 \sqrt{\ln s}+4 \phi_{n}(s)\right) \frac{\mathrm{ds}}{\mathrm{s}}, n \geq 0, \\
\omega_{n+1}(s) & =\mathbb{E}_{0.5,1}\left(-4 \sqrt{\ln \frac{\varsigma}{s}}\right)+\int_{1}^{\varsigma}\left(\ln \frac{\varsigma}{s}\right)^{-0.5} \mathbb{E}_{0.5,0.5}\left(-4 \sqrt{\ln \frac{\varsigma}{s}}\right) \\
& \times\left(1-\omega_{n}^{2}(s)+2 \sqrt{\ln s}+4 \omega_{n}(s)\right) \frac{\mathrm{ds}}{\mathrm{s}}, n \geq 0 .
\end{aligned}
$$

Example 2. Consider the following Caputo FDE

$$
\left\{\begin{array}{l}
{ }^{c} \mathbb{D}_{0^{+}}^{v} \phi(\varsigma)=\sin (\phi(\varsigma))-\phi(\varsigma), \varsigma \in[0,1], \\
0.5 \phi(0)-3 \phi(0) \phi(1)=0 .
\end{array}\right.
$$

Note that problem (12) is a particular case of problem (1), where

$$
v=0.5, a=0, b=1, \vartheta(\varsigma)=\varsigma,
$$

and

$$
\left\{\begin{array}{l}
\mathbb{F}(\varsigma, \phi(\varsigma))=\sin (\phi(\varsigma))-\phi(\varsigma), \quad \varsigma \in \mathrm{J}, \\
\mathbb{G}(\phi(0), \phi(1))=0.5 \phi(0)-3 \phi(0) \phi(1)
\end{array}\right.
$$

Taking $\phi_{0}(\varsigma)=0$ and $\omega_{0}(\varsigma)=\sqrt{\varsigma}$, it is easy to verify that $\phi_{0}, \omega_{0}$ are lower and upper solutions of (12), respectively, and $\phi_{0}(\varsigma) \leq \omega_{0}(\varsigma)$, for $\varsigma \in[0,1]$. Therefore, Hypothesis 1 of Theorem 1 holds. However, if $\phi_{0}(\varsigma) \leq \mathrm{x} \leq \mathrm{y} \leq \omega_{0}(\varsigma)$ we have

$$
\mathbb{F}(\varsigma, y)-\mathbb{F}(\varsigma, \mathrm{x}) \geq-2(\mathrm{y}-\mathrm{x}), \quad \varsigma \in[0,1] .
$$

Hence Hypothesis 2 holds with $\lambda=2$, and if $\phi_{0}(0) \leq u_{1} \leq u_{2} \leq \omega_{0}(0), \phi_{0}(1) \leq v_{1} \leq$ $v_{2} \leq \omega_{0}(1)$, we have

$$
\mathbb{G}\left(u_{2}, v_{2}\right)-\mathbb{G}\left(u_{1}, v_{1}\right) \leq\left(u_{2}-u_{1}\right) .
$$

Therefore, Hypothesis 3 holds with $c=1$ and $d=0$. According to Theorem 1, there exist monotone iterative sequences $\left\{\phi_{n}\right\}$ and $\left\{\omega_{n}\right\}$ that are uniformly converging to $\phi^{*}$ and $\omega^{*}$, respectively, and $\phi^{*}, \omega^{*}$ are the extremal solutions in $\left[\phi_{0}, \omega_{0}\right]$ of problem (12).

Example 3. Consider the following $\vartheta$-Caputo FDE

$$
\left\{\begin{array}{l}
{ }^{c} \mathbb{D}_{0^{+}}^{0.5 ; e^{2 \varsigma}} \phi(\varsigma)=\frac{2}{\sqrt{\pi}} \sqrt{e^{2 \varsigma}-1}+e^{2 \varsigma}-1-\sin \left(e^{2 \varsigma}-1\right)+\sin (\phi(\varsigma))-\phi(\varsigma), \varsigma \in[0,1], \\
\phi(0)=\frac{2 \pi}{e^{2}-1+2 \pi} \phi(1) .
\end{array}\right.
$$


Comparing the above problem with problem (1), we obtain

$$
v=0.5, a=0, b=1, \vartheta(\varsigma)=e^{2 \varsigma},
$$

and

$$
\left\{\begin{array}{l}
\mathbb{F}(\varsigma, \phi(\varsigma))=\frac{2}{\sqrt{\pi}} \sqrt{e^{2 \varsigma-1}}+e^{2 \varsigma}-1-\sin \left(e^{2 \varsigma}-1\right)+\sin (\phi(\varsigma))-\phi(\varsigma), \quad \varsigma \in \mathrm{J}, \\
\mathbb{G}(\phi(0), \phi(1))=\phi(0)-\frac{2 \pi}{e^{2}-1+2 \pi} \phi(1) .
\end{array}\right.
$$

One can verify that $\phi(\varsigma)=e^{2 \varsigma}-1$ is an exact solution of the problem (1). Moreover, taking $\phi_{0}(\varsigma)=0$ and $\omega_{0}(\varsigma)=e^{2 \varsigma}-1+2 \pi$, it is easy to verify that $\phi_{0}, \omega_{0}$ are lower and upper solutions of (13), respectively, and $\phi_{0}(\varsigma) \leq \omega_{0}(\varsigma)$, for $\varsigma \in[0,1]$. Therefore, Hypothesis 1 of Theorem 1 holds. However, if $\phi_{0}(\varsigma) \leq \mathrm{x} \leq \mathrm{y} \leq \omega_{0}(\varsigma)$ we have

$$
\mathbb{F}(\zeta, y)-\mathbb{F}(\varsigma, \mathrm{x}) \geq-2(\mathrm{y}-\mathrm{x}), \quad \varsigma \in[0,1] .
$$

Hence Hypothesis 2 holds with $\lambda=2$, and if $\phi_{0}(0) \leq u_{1} \leq u_{2} \leq \omega_{0}(0), \phi_{0}(1) \leq v_{1} \leq$ $v_{2} \leq \omega_{0}(1)$, we have

$$
\mathbb{G}\left(u_{2}, v_{2}\right)-\mathbb{G}\left(u_{1}, v_{1}\right) \leq\left(u_{2}-u_{1}\right)-\frac{2 \pi}{e^{2}-1+2 \pi}\left(v_{2}-v_{1}\right)
$$

Therefore, Hypothesis 3 holds with $c=1$ and $d=\frac{2 \pi}{e^{2}-1+2 \pi}$. According to Theorem 1, there exist monotone iterative sequences $\left\{\phi_{n}\right\}$ and $\left\{\omega_{n}\right\}$ that are uniformly converging to $\phi^{*}$ and $\omega^{*}$, respectively, and $\phi^{*}, \omega^{*}$ are the extremal solutions in $\left[\phi_{0}, \omega_{0}\right]$ of problem (13).

\section{Conclusions}

We have established sufficient results by using monotone iterative techniques together with upper and lower solutions for a class of boundary value problem involving a generalized form of Caputo derivative of fractional order. By using the mentioned tool, we have established fruitful combinations between lower and upper solutions. Further, the said method has the ability to produce two sequences of upper and lower solutions, respectively. For the construction of the aforesaid sequences this method does not need any kind of discretization or collocation like other methods usually require. The two sequences we have generated are of a monotonic type with increasing and decreasing behaviors, respectively. Moreover, the sequence that is monotonically decreasing converges to its lower bound. However, the other one that is monotonically increasing is converging to its upper bound. The bounds for upper and lower solutions have also been investigated for their uniqueness using Banach contraction theorem. For the justification of our results, we have provided some examples. Overall we have concluded that the proposed procedure is a powerful and efficient tool to study various classes of FDEs for their extremal solutions. In future this technique can be applied to investigate those classes of FDEs involving non-singular-type derivatives under boundary conditions for upper and lower solution. In addition, the mentioned tool can be applied to investigate fractal-fractional-type problems corresponding to boundary conditions. Overall we have concluded that the monotone iterative technique of applied analysis is a powerful technique for dealing with various kinds of problems involving different types of fractional-order operators. 


\begin{abstract}
Author Contributions: Conceptualization, C.D., Z.B.; writing-original draft preparation, C.D., Z.B. and M.S.A.; methodology, C.D. and Z.B.; software, K.S. and B.A.; validation, C.D., Z.B., M.S.A. and T.A.; formal analysis, C.D., Z.B. and M.S.A.; writing-review and editing, C.D., Z.B., M.S.A., K.S. and T.A.; investigation, M.S.A., K.S., B.A. and T.A. All authors have read and agreed to the published version of the manuscript.
\end{abstract}

Funding: This work was funded by the Prince Sultan University, Riyadh P.O. Box 11586, Saudi Arabia. Institutional Review Board Statement: Not applicable.

Informed Consent Statement: Not applicable.

Data Availability Statement: Not applicable.

Acknowledgments: Authors Kamal Shah, Bahaaeldin Abdalla and Thabet Abdeljawad would like to thank Prince Sultan University for paying the APC and support through TAS research lab.

Conflicts of Interest: The authors declare no conflicts of interest.

\title{
References
}

1. Hilfer, R. Applications of Fractional Calculus in Physics; World Scientific: Singapore, 2000.

2. Mainardi, F. Fractional Calculus and Waves in Linear Viscoelasticity; Imperial College Press: London, UK, 2010.

3. Tarasov, V.E. Fractional Dynamics; Nonlinear Physical Science; Springer: Heidelberg, Germany, 2010.

4. Abbas, S.; Benchohra, M.; Graef, J.R.; Henderson, J. Implicit Fractional Differential and Integral Equations: Existence and Stability; De Gruyter: Berlin, Germany, 2018.

5. Diethelm, K. The Analysis of Fractional Differential Equations: An Application-Oriented Exposition Using Differential Operators of Caputo Type; Springer Science \& Business Media: New York, NY, USA, 2010.

6. Kilbas, A.A.; Srivastava, H.M.; Trujillo, J.J. Theory and Applications of Fractional Differential Equations; North-Holland Mathematics Sudies; Elsevier: Amsterdam, The Netherlands, 2006; Volume 204.

7. Gorenflo, R.; Kilbas, A.A.; Mainardi, F.; Rogosin, S.V. Mittag-Leffler Functions, Related Topics and Applications; Springer: New York, NY, USA, 2014.

8. Tenreiro Machado, J.A.; Silva, M.F.; Barbosa, R.S.; Jesus, I.S.; Reis, C.M.; Marcos, M.G.; Galhano, A.F. Some applications of fractional calculus in engineering. Math. Probl. Eng. 2010, 34, 639801. [CrossRef]

9. da Graça Marcos, M.; Duarte, F.B.M.; Machado, J.A.T. Complex dynamics in the trajectory control of redundant manipulators Nonlinear Sci. Complex. 2007, 2007, 134143.

10. Atangana, A.; Kilicman, A. On the generalized mass transport equation to the concept of variable fractional derivative. Math. Probl. Eng. 2014, 2014, 542809. [CrossRef]

11. Almeida, R. A Caputo fractional derivative of a function with respect to another function. Commun. Nonlinear Sci. Numer. Simul. 2017, 44, 460-481. [CrossRef]

12. Vanterler da C. Sousa, J.; Capelas de Oliveira, E. On the $\psi$-Hilfer fractional derivative. Commun. Nonlinear Sci. Numer. Simul. 2018, 60, 72-91. [CrossRef]

13. Sousa, J.V.D.C.; de Oliveira, E.C. On the Stability of a Hyperbolic Fractional Partial Differential Equation. Differ. Equ. Dyn. Syst. 2019, 2019, 730465. [CrossRef]

14. Du, S.W.; Lakshmikantham, V. Monotone iterative technique for differential equations in a Banach space. J. Math. Anal. Appl. 1982, 87, 454-459. [CrossRef]

15. Guo, D.; Lakshmikantham, V. Nonlinear Problems in Abstract Spaces; Academic Press: New York, NY, USA, 1988.

16. Derbazi, C.; Baitiche, Z. Coupled systems of $\psi$-Caputo differential equations with initial conditions in Banach spaces. Mediterr. J. Math. 2020, 17, 169. [CrossRef]

17. Derbazi, C.; Baitiche, Z.; Fečkan, M. Some new uniqueness and Ulam stability results for a class of multiterms fractional differential equations in the framework of generalized Caputo fractional derivative using the $\Phi$-fractional Bielecki-type norm. Turkish J. Math. 2021, 45, 2307-2322. [CrossRef]

18. Derbazi, C.; Baitiche, Z.; Benchohra, M.; N'Guérékata, G. Existence, uniqueness, and Mittag-Leffler-Ulam stability results for Cauchy problem involving $\psi$-Caputo derivative in Banach and Fréchet spaces. Int. J. Differ. Equ. 2020, 2020, 6383916. [CrossRef]

19. Baitiche, Z.; Guerbati, K.; Benchohra, M.; Zhou, Y. Solvability of Fractional Multi-Point BVP with Nonlinear Growth at Resonance. J. Contemp. Math. Anal. 2020, 55, 126-142. [CrossRef]

20. Benchohra, M.; Bouriah, S.; Nieto, J.J. Existence of periodic solutions for nonlinear implicit Hadamard's fractional differential equations. Rev. R. Acad. Cienc. Exactas Fís. Nat. Ser. A Mat. 2018, 112, 25-35. [CrossRef]

21. Ladde, G.S.; Lakshmikantham, V.; Vatsala, A.S. Monotone Iterative Techniques for Nonlinear Differential Equations; Pitman Publishing: Bay City, MI, USA, 1985; Volume 27.

22. Heikkilä, S.; Lakshmikantham, V. Monotone Iterative Techniques for Discontinuous Nonlinear Differential Equations; Taylor \& Francis: New York, NY, USA, 2017. 
23. Hristova, S.S.; Bainov, D.D. Monotone-iterative techniques of V. Lakshmikantham for a boundary value problem for systems of impulsive differential-difference equations. J. Nath. Anal. Appl. 1996, 197, 1-13. [CrossRef]

24. Bhaskar, T.G.; Farzana, A.M. Monotone iterative techniques for nonlinear problems involving the difference of two monotone functions. Appl. Math. Comput. 2002, 133, 187-192. [CrossRef]

25. Lakshmikantham, V.; Vatsala, A.S. General uniqueness and monotone iterative technique for fractional differential equations. Appl. Math. Lett. 2008, 21, 828-834. [CrossRef]

26. Franco, D.; Nieto, J.J.; O'Regan, D. Existence of solutions for first order ordinary differential equations with nonlinear boundary conditions. Appl. Math. Comput. 2004, 153, 793-802. [CrossRef]

27. Lin, X.; Zhao, Z. Iterative technique for a third-order differential equation with three-point nonlinear boundary value conditions. Electron. J. Qual. Theory Differ. Equ. 2016, 2016, 1-10. [CrossRef]

28. West, I.H.; Vatsala, A.S. Generalized monotone iterative method for initial value problems. Appl. Math. Lett. 2004, 17, 1231-1237. [CrossRef]

29. Xu, H.K.; Nieto, J.J. Extremal solutions of a class of nonlinear integro-differential equations in Banach spaces. Proc. Amer. Math Soc. 1997, 125, 2605-2614. [CrossRef]

30. Al-Refai, M.; Ali Hajji, M. Monotone iterative sequences for nonlinear boundary value problems of fractional order. Nonlinear Anal. 2011, 74, 3531-3539. [CrossRef]

31. Derbazi, C.; Baitiche, Z.; Benchohra, M.; Cabada, A. Initial value problem for nonlinear fractional differential equations with $\psi$-Caputo derivative via monotone iterative technique. Axioms 2020, 9, 57. [CrossRef]

32. Derbazi, C.; Baitiche, Z.; Benchohra, M.; N'Guérékata, G. Existence, uniqueness, approximation of solutions and $E_{\alpha}$-Ulam stability results for a class of nonlinear fractional differential equations involving $\psi$-Caputo derivative with initial conditions. Math. Morav. 2021, 25, 1-30. [CrossRef]

33. Baitiche, Z.; Derbazi, C.; Alzabut, J.; Samei, M.E.; Kaabar, M.K.A.; Siri, Z. Monotone Iterative Method for $\vartheta$-Caputo Fractional Differential Equation with Nonlinear Boundary Conditions. Fractal Fract. 2021, 5, 81. [CrossRef]

34. Baitiche, Z.; Derbazi, C.; Wang, G. Monotone iterative method for nonlinear fractional p-Laplacian differential equation in terms of $\psi$-Caputo fractional derivative equipped with a new class of nonlinear boundary conditions. Math. Meth. Appl. Sci. 2021, 45, 967-976. [CrossRef]

35. Wang, G. Monotone iterative technique for boundary value problems of a nonlinear fractional differential equation with deviating arguments. J. Comput. Appl. Math. 2012, 236, 2425-2430. [CrossRef]

36. Wang, G.; Sudsutad, W.; Zhang, L.; Tariboon, J. Monotone iterative technique for a nonlinear fractional $q$-difference equation of Caputo type. Adv. Difference Equ. 2016, 211, 11. [CrossRef]

37. Zhang, S. Monotone iterative method for initial value problem involving Riemann-Liouville fractional derivatives. Nonlinear Anal. 2009, 71, 2087-2093. [CrossRef]

38. Abdo, M.S.; Ibrahim, A.G.; Panchal, S.K. Nonlinear implicit fractional differential equation involving-Caputo fractional derivative, Proc. Jangjeon Math. Soc. 2019, 22,387-400.

39. Patil, J.; Chaudhari, A.; Abdo, M.S.; Hardan, B. Upper and lower solution method for positive solution of generalized Caputo fractional differential equations. Adv. Theo. Nonl. Anal. Appl. 2020, 4, 279-291. [CrossRef]

40. Wahash, H.A.; Panchal, S.K.; Abdo, M.S. Positive solutions for generalized Caputo fractional differential equations with integral boundary conditions. J. Math. Model. 2020, 8, 393-414.

41. Wei, Z.; Li, Q.; Che, J. Initial value problems for fractional differential equations involving Riemann-Liouville sequential fractional derivative. J. Math. Anal. Appl. 2010, 367, 260-272. [CrossRef] 